\title{
Desempenho acústico de paredes de blocos e tijolos cerâmico: uma comparação entre Brasil e Portugal
}

\author{
Acoustic performance of ceramic brick and block \\ walls: a comparative study between Brazil and \\ Portugal
}

\section{Maria de Fatima Ferreira Neto Stelamaris Rolla Bertoli}

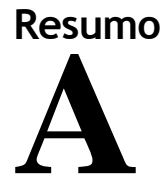

lguns questionamentos sobre a relação entre desempenho acústico e o conforto acústico percebido contribuíram para o início desta pesquisa, que estuda o desempenho e conforto acústicos de ambientes compostos por blocos e tijolos cerâmicos, respectivamente no Brasil e em Portugal. Os tijolos portugueses apresentam 11,0 cm e 15,0 cm de espessura, enquanto os blocos brasileiros $11,5 \mathrm{~cm}$ e $14,0 \mathrm{~cm}$ de espessura. A avaliação do comportamento acústico desses elementos consistiu de testes em laboratório e em campo. Os critérios de desempenho utilizados para a avaliação do desempenho acústico foram a norma ABNT NBR 15575, do Brasil, e o Decreto-lei n 96/2008, de Portugal. A avaliação do conforto acústico consistiu na análise da participação de um júri, que indicou a percepção de sentenças emitidas por vozes feminina e masculina, em simulação de convivência entre vizinhos adjacentes. Os resultados do desempenho acústico dessas paredes estão aquém do recomendado pelos critérios locais para o uso como parede de geminação. A avaliação dos resultados com o júri corroborou essa conclusão. Quanto ao desempenho acústico, verifica-se que a indústria da construção civil destes dois países deve aprimorar-se para atender aos critérios da nova norma.

Maria de Fatima Ferreira Neto

Departamento de Arquitetura e Construção, Faculdade de Engenharia Civil, Arquitetura e Urbanismo Universidade Estadual de Campinas

Av. Albert Einstein, 951, Caix Postal 6021

Campinas - SP - Brasil CEP 13083-852

Tel.: (19) 3521-2382

E-mail: neto.fis@hotmail.com

Stelamaris Rolla Bertoli Departamento de Arquitetura e Construção, Faculdade de Engenharia Civil, Arquitetura e Urbanismo

Universidade Estadual de Campinas

Tel.: (19) 3521-2382

E-mail: rolla@fec.unicamp.br

Recebido em 22/02/10 Aceito em 20/10/10
Palavras-chave: Desempenho acústico. Isolamento acústico. Conforto acústico. Paredes de alvenaria.

\section{Abstract}

Some questions about the relationship between acoustical performance and perceived acoustic comfort contributed to the start of this research study, which investigated the performance and acoustic comfort in buildings that have ceramic block and brick walls, respectively, in Brazil and Portugal. Portuguese bricks are $11.0 \mathrm{~cm}$ and $15.0 \mathrm{~cm}$ thick, and the Brazilian blocks are $11.5 \mathrm{~cm}$ and $14.0 \mathrm{~cm}$ thick. The evaluation of the acoustic performance of those elements consisted of laboratory and field experiments. The performance criteria used to evaluate the acoustic performance was the Brazilian standard NBR 15575 and the Portuguese law 96/2008. The evaluation of acoustic comfort consisted of the analysis of the participation of a jury, which indicated the perception of sentences produced by male and female voices, in a simulation of coexistence between adjacent neighbours. The results of the acoustic performance of those walls are below what is recommended by local criteria for their use as twinning walls. The evaluation of the results by the jury supports that conclusion. The study concludes that the building industries in those two countries need to improve acoustic performance in order to meet the requirements of new standards.

Keywords: Acoustic performance. Sound insulation. Acoustical comfort. Masonry walls. 


\section{Introdução}

Blocos ou tijolos cerâmicos são comumente utilizados em edificações tanto no Brasil quanto em Portugal. Cada um dos países define seus padrões próprios em dimensão, massa, resistência, por meio de suas normas, regulamentos e especificações próprias. Devido às diferenças nas definições de bloco e tijolo entre os dois países, neste trabalho, o uso da palavra "bloco" será referente ao utilizado no Brasil e de "tijolo", referente a Portugal.

Em Portugal, o uso do tijolo cerâmico como material na construção é muito antigo, juntamente com a pedra e a madeira. $\mathrm{O}$ uso do tijolo cerâmico industrializado, com furação horizontal, em construção vem desde o século XIX. Em 1964, o Instituto Português da Qualidade (IPQ) apresentou a norma portuguesa NP 80, na qual define as características e ensaios de tijolos para alvenaria (ASSOCIAÇÃO..., 2000). No Brasil, o uso do tijolo cerâmico também é bastante comum e antigo. Em 1940, a Associação Brasileira de Normas Técnicas (ABNT) aprovou normas especificando a produção do tijolo de barro cozido maciço com comprimento de $22,0 \mathrm{~cm}$, largura de $11,0 \mathrm{~cm}$ e altura de $5,5 \mathrm{~cm}$. Posteriormente, surgiu o tijolo furado de $20,0 \mathrm{~cm}$ x $20,0 \mathrm{~cm}$. O bloco de concreto de $20,0 \mathrm{~cm}$ x 40,0 $\mathrm{cm}$ veio logo na sequência (ABCI, 1990).

Independentemente do tipo de tijolo ou bloco utilizado na construção de edifícios residenciais, os usuários desejam encontrar conforto dentro de suas moradias. O conforto acústico é um dos almejados. Segundo Barry (2008), um recinto de uma edificação deve proporcionar ao usuário conforto e privacidade acústica condizentes à finalidade do ambiente, sobretudo quando se destina ao repouso ou trabalho intelectual. Entre os itens que causam maior desconforto acústico aos moradores, aparecem a conversação de vizinhos, o impacto no piso, causado por caminhamento, e a queda de objetos, latidos de cães e brincadeiras de crianças. Grimwood (1997) mencionou em seu trabalho que seus entrevistados, moradores de edifícios residenciais, sentem-se prejudicados com o baixo desempenho das paredes em relação ao ruído aéreo, e também com o baixo desempenho do piso em relação ao impacto. A vida social passa a ser afetada, pois as pessoas deixam de receber ou fazer visitas, para evitar que o ruído produzido incomode os vizinhos, ou prejudique o próprio ambiente. Em 1988, Utley e Buller afirmaram, em trabalho publicado no Journal of Sound and Vibration, que o ruído da vizinhança (vizinhos, crianças e animais) é a fonte de ruído que causa mais desconforto, perdendo apenas para o ruído do tráfego rodoviário. O desconforto causado por ruídos provenientes da vizinhança é um assunto antigo. Em 1955, Bitter e Van Veeren $\left(1955^{1}\right.$ apud CROOME, 1977) mostraram em seu trabalho que os ruídos que causam mais desconforto são impacto de portas em batida, quedas de objetos, caminhamento e impactos em pisos. Ruídos de crianças brincando, rádio, TV ou conversação causam um desconforto moderado.

O cuidado para evitar que o nível de ruído incomode a vizinhança tem de ser redobrado, bem como com que o ruído emitido pela vizinhança chegue a sua residência em níveis considerados desconfortáveis. Esse incômodo e esse desconforto refletem a necessidade de haver um bom isolamento acústico entre unidades residenciais e entre ambientes internos da mesma unidade residencial. Segundo Barry (2005), o usuário não está interessado, prioritariamente, na quantidade de isolamento, mas sim no nível de ruído resultante e espera que um recinto de uma edificação lhe proporcione conforto e privacidade acústicos. Em se tratando de pesquisas sobre isolamento acústico, inúmeros são os trabalhos relacionados ao desempenho acústico de materiais utilizados na construção civil. Alguns trabalhos tratam do desempenho da alvenaria (PAIXÃO, 2002), outros tratam da eficiência das janelas com vidros simples ou vidros duplos (COLLINS et al., 1995). A preocupação com o conforto acústico também foi tratada por Fuchs et al. (2001), de um modo particular com as baixas frequências. Entre os trabalhos de isolamento sonoro em edificações pode-se citar os trabalhos de Gerretsen (2003a, 2003b), em que apresenta os valores definidos em normas específicas a que a partição deve obedecer, e o trabalho de Lahtela (2005), que apresenta um guia de isolamento sonoro em edificações de madeira, comuns na Finlândia. Nesse trabalho, o autor também teve o cuidado de mostrar os valores mínimos de isolamento estabelecidos em documentos que a edificação deve ter.

Em relação à privacidade, Cavanaugh et al., em 1962, já questionava sobre os valores de isolamento dos materiais obtidos na época. Insistia na realização de testes de laboratório (embora ainda não houvesse normas específicas para a avaliação). Afirmava, ainda, que o nível de privacidade dependia das atividades a serem executadas. Por essa razão, foram realizados diversos estudos sobre a privacidade da fala em vários ambientes, entre eles residências, escritórios e hotéis.

${ }^{1}$ BITTER, C.; VAN WEEREN, P. Report 24, Research Institute for Public Health Engineering, TNO. Holland: The Hague, 1955. 


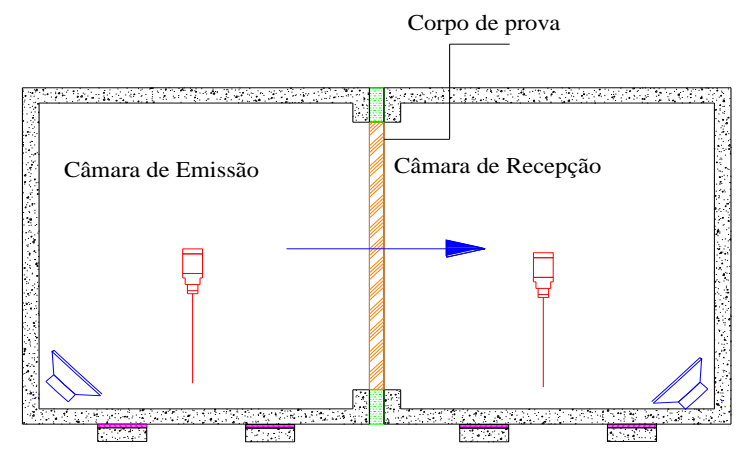

Fonte: Adaptado de Barry (2005).

Figura 1 - Desenho esquemático de câmaras reverberantes em teste de isolação sonora, em laboratório

O objetivo deste trabalho é apresentar a comparação de desempenho acústico de dois grupos de paredes de alvenaria, sendo o Grupo I paredes compostas de tijolos/blocos com espessuras de $11,0 \mathrm{~cm}$ e $11,5 \mathrm{~cm}$, e o Grupo II, tijolos/blocos com espessuras de 15,0 cm e 14,0 $\mathrm{cm}$. Essas paredes, com a finalidade de geminação, isto é, entre duas unidades habitacionais, são utilizadas em edifícios residenciais em Portugal e no Brasil. Essa avaliação do desempenho envolve testes realizados em laboratório e em campo, e teste de percepção da inteligibilidade de sentenças, com júri.

\section{Método}

Para realizar a avaliação do desempenho acústico, as paredes de alvenaria foram submetidas ao teste de isolação ${ }^{2}$ sonora em laboratório e isolamento ${ }^{3}$ sonoro em campo. Esses testes seguiram os procedimentos das normas ISO 140-3 (1995) para o laboratório e ISO 140-4 (1998) para o campo. A norma ISO 717-1 (1996) foi utilizada para obter o número único de desempenho.

Os procedimentos de medição para o laboratório e para o campo são similares. A parede em avaliação (corpo de prova) fica entre dois ambientes, sendo um de emissão e outro de recepção, conforme esquematizado na Figura 1.

No ambiente de emissão, é medido o nível de pressão sonora de ruído branco. ${ }^{4}$ No ambiente de

\footnotetext{
${ }^{2}$ Isolação - refere-se ao fenômeno acústico. A isolação é avaliada em laboratório e refere-se ao comportamento acústico de um único elemento construtivo isoladamente (BARING, 1988). A avaliação provém da relação entre a energia sonora incidente e a energia sonora transmitida pelo corpo de prova.

${ }^{3}$ Isolamento - refere-se à redução do som entre dois recintos, proporcionado pelo elemento construtivo entre eles, que pode ser a fachada, parede ou piso. É a capacidade de um ambiente de ser protegido de ruídos externos ou de confinar fontes de ruído (BARING, 1988).

${ }^{4}$ Ruído branco - seu espectro apresenta nível constante em todas as frequências (BISTAFA, 2006).
}

recepção, são medidos o nível de pressão sonora do ruído branco após a passagem pela parede, o nível de pressão sonora do ruído de fundo ${ }^{5}$ e o tempo de reverberação. ${ }^{6}$ Em laboratório, as condições ambientais são controladas, e parâmetros como umidade do ar e temperatura são medidos e entram no cálculo (embutidos no cálculo da absorção sonora no ambiente de recepção, A) do Índice de Redução Sonora, R, como mostra a Equação 1.

$\mathrm{R}=\mathrm{L}_{1}-\mathrm{L}_{2}+10 \log \left(\frac{\mathrm{S}}{\mathrm{A}}\right)$

Onde:

$\mathrm{L}_{1}$ - nível médio no ambiente de emissão, em dB;

$\mathrm{L}_{2}$ - nível médio no ambiente de recepção, em dB;

$\mathrm{S}$ - área do corpo de prova, em $\mathrm{m}^{2}$; e

A - absorção sonora no ambiente de recepção, em $\mathrm{m}^{2}$.

Em campo, esses parâmetros não são controlados e não entram no cálculo da Diferença Padronizada de Nível, $\mathrm{D}_{\mathrm{nT}}$, dada pela Equação 2.

$\mathrm{D}_{\mathrm{nT}}=\mathrm{L}_{1}-\mathrm{L}_{2}+10 \log \left(\frac{\mathrm{T}}{\mathrm{T}_{\mathrm{o}}}\right)$

Eq. 2

Onde:

$\mathrm{L}_{1}$ - nível médio no ambiente de emissão, em dB;

$\mathrm{L}_{2}$ - nível médio no ambiente de recepção, em dB;

$\mathrm{T}$ - tempo de reverberação no ambiente de recepção, em segundos; e

$\mathrm{T}_{\mathrm{o}}$ - tempo de reverberação de referência, em edificações, $\mathrm{T}_{\mathrm{o}}=0,5 \mathrm{~s}$.

\footnotetext{
${ }^{5}$ Ruído de fundo - ruído fontes secundárias que se sobrepõem ao ruído principal (BISTAFA, 2006).

${ }^{6}$ Tempo de reverberação - tempo necessário para que o nível em um recinto caia $60 \mathrm{~dB}$ após o desligamento da fonte sonora (BISTAFA, 2006).
} 
Como se verifica pela Equação 2, para o cálculo da Diferença Padronizada de Nível não é necessário inserir o volume do ambiente de recepção, visto que está embutido nos resultados da medição do tempo de reverberação.

Em laboratório foram utilizados os seguintes equipamentos: uma fonte sonora omnidirecional, um analisador de ruído de dois canais (porém, foi utilizado somente um canal para a medição), um microfone de 1/2", pré-amplificador para o microfone e amplificador para o gerador de sinais.

Ainda em laboratório, para a medição de níveis de ruído branco, tanto no ambiente de emissão quanto no ambiente de recepção, foram utilizadas uma posição de fonte sonora, posicionada em um triedro inferior da câmara de emissão, e seis posições de microfone. O cálculo dos níveis de ruído é obtido a partir das médias, espacial e temporal, das medições. Para a medição do tempo de reverberação, o método utilizado foi a resposta impulsiva integrada com excitação por MLS (maximum-length sequences). Foram utilizadas duas posições de caixas acústicas colocadas em dois dos triedros inferiores da câmara reverberante e seis posições do microfone. A medição do tempo de reverberação se faz segundo os procedimentos da norma ISO 354 (2003). O ruído de fundo também foi medido em seis posições do microfone. A avaliação em laboratório, em Portugal, foi realizada por Pião (2007).

Para as medições em campo realizadas no Brasil, foram utilizados os seguintes instrumentos: gerador de sinais e analisador de ruído de dois canais, pré-amplificador do microfone e microfone de $1 / 2$ ", uma fonte sonora omnidirecional e amplificador de potência. Esses equipamentos são de propriedade da Unicamp. Foram utilizadas quatro posições de microfone no ambiente de recepção. As normas de procedimento de medição estipulam distâncias mínimas entre a fonte e o microfone $\mathrm{e}$ entre $\mathrm{o}$ microfone $\mathrm{e}$ as demais superfícies. Conforme essas distâncias e a área do ambiente de recepção, pode-se variar o número de posições de microfone. O número de posições de fonte sonora foi o mesmo do usado em laboratório.

Em Portugal, os instrumentos utilizados, pertencentes à Faculdade de Ciências e Tecnologia da Universidade de Coimbra, foram: analisador de ruído de dois canais, gerador de ruído independente do analisador, amplificador de potência para o gerador de ruído, pré-amplificador para o microfone e microfone de $1 / 2$ ", condicionador de sinal e fonte sonora omnidirecional. Os números de posições de microfone e fonte seguiram os mesmos números da medição realizada no Brasil.
Nas medições dos níveis de pressão sonora do ruído em campo, no Brasil e em Portugal, foi utilizado o ruído branco, conforme a norma ISO 140-4 (1998). O tempo de reverberação foi medido com o ruído rosa, ${ }^{7}$ segundo os procedimentos da norma ISO 354 (2003).

A obtenção do número único é feita por meio da ponderação de uma curva de referência dada pela norma ISO 717-1 (1996) com a curva do desempenho, seja $\mathrm{R}$ ou $\mathrm{D}_{\mathrm{nT}}$. A curva de referência colocada sobre a curva de desempenho deve ser deslocada verticalmente, até que a soma das diferenças entre a curva de referência e a de desempenho de cada uma das frequências, em bandas de $1 / 3$ de oitava, entre $100 \mathrm{~Hz}$ e $3.150 \mathrm{~Hz}$, seja igual ou inferior a 32,0. Definida a nova posição da curva de referência, obtém-se o valor de $\mathrm{R}_{\mathrm{w}}$ (Índice de Redução Sonora Ponderado) ou $\mathrm{D}_{\mathrm{nT}, \mathrm{w}}$ (Diferença Padronizada de Nível Ponderada) pelo valor da curva de referência deslocada, em $500 \mathrm{~Hz}$.

Também em campo, foi feita uma avaliação da parede, por um júri, em relação à percepção da inteligibilidade de sentenças emitidas por vozes masculina e feminina, com procedimento criado pelas autoras, visto que até então a inteligibilidade da fala é avaliada em um único ambiente, e não entre dois ambientes com uma partição entre eles. Esse procedimento consistiu na emissão de grupo de 10 sentenças por vozes feminina e masculina, por meio de um sistema sonoro, com nível de pressão sonora médio de $85 \mathrm{~dB}$, no ambiente chamado de emissão. Tais sentenças foram gravadas em um estúdio nas dependências da Unicamp, em Campinas, por voz masculina e voz feminina. Embora o nível de conversação normal esteja entre $60 \mathrm{~dB}$ e $70 \mathrm{~dB}$ (HASSAL; ZAVERI, 1988), esse nível de 85 dB (sem ponderação) foi escolhido por representar um nível audível no ambiente de recepção (nível definido após simulações com voz ao vivo no ambiente de emissão e o júri no ambiente de recepção), em representação à conversação, mesmo com nível acima do normal, bem como aparelhos de som, TV e rádio quando em funcionamento. Abaixo desse nível havia dificuldade de grau elevado na percepção das sentenças, o que colocaria em questão o resultado subjetivo com o júri.

O júri, composto de pessoas com audição não comprometida, posicionado no ambiente de recepção, responde sobre a percepção do sinal sonoro, no caso, das sentenças ouvidas. $\mathrm{O}$ conjunto de sentenças utilizadas na avaliação subjetiva foi

\footnotetext{
${ }^{7}$ Ruído rosa - seu espectro apresenta nível que cai $3 \mathrm{~dB}$ por oitava (BISTAFA, 2006).
} 
baseado nas sentenças utilizadas em testes audiométricos (GAMBINI, 2006).

Tanto no Brasil quanto em Portugal, o auxílio do júri se faz necessário para a avaliação subjetiva do desempenho acústico da parede. Para essa avaliação em Portugal, embora o povo português esteja muito familiarizado com a pronúncia brasileira devido ao grande número de programas brasileiros sem dublagem, que são transmitidos nos canais portugueses de televisão, houve o cuidado de selecionar pessoas que já tiveram contato com a língua no Brasil. Nessa avaliação, o júri foi posicionado no ambiente de recepção, simulando os moradores de uma unidade habitacional em um edifício residencial.

Nível de privacidade e nível de conforto acústico são parâmetros criados no trabalho de Neto (2009) a partir de análise mais detalhada do desempenho de diversos sistemas construtivos e da inteligibilidade da fala no ambiente de recepção. A privacidade e o conforto acústico são definições diferentes do desempenho acústico que uma parede ou ambiente podem apresentar. A partir do bom desempenho, pode-se obter a privacidade e o conforto acústico almejados, desde que as condições acústicas do ambiente de recepção também sejam favoráveis. Neste trabalho não foi meta a justificativa dos resultados do desempenho com a percepção das sentenças pelo júri, utilizando essa diferença de definição de conforto e desempenho e ainda o uso dos parâmetros psicoacústicos. Em trabalhos futuros serão publicadas a relação do desempenho com a percepção subjetiva e a apresentação desses novos parâmetros de análise de conforto. Alguns dos resultados inesperados aqui atingidos poderão ser clarificados nesses trabalhos. Com este presente trabalho mostra-se que há um problema desempenho da parede que até pode atender à norma NBR 15575 (ABNT, 2008), porém permite que se escutem conversações no ambiente adjacente; no caso, apartamento vizinho - e há uma análise inicial - avaliação desse desempenho e a opinião de um júri -, que, embora tenham parâmetros que possam auxiliar na justificativa dos resultados, não são aqui estudados (parâmetros psicoacústicos), por necessitarem de uma análise aprofundada, o que virá futuramente.

\section{Paredes avaliadas}

As paredes avaliadas são compostas de blocos e tijolos cerâmicos. No Brasil, os prédios onde foram realizadas as avaliações em campo eram prédios novos, prontos para serem habitados. Em Portugal, o prédio já estava habitado e foi construído no início dos anos 90. As paredes avaliadas foram separadas em dois grupos, como mencionado, sendo o Grupo I referente às paredes com $11,0 \mathrm{~cm}$ e $11,5 \mathrm{~cm}$, e o Grupo II, às paredes com $14,0 \mathrm{~cm}$ e $15,0 \mathrm{~cm}$. As paredes portuguesas eram compostas de tijolos de $11,0 \mathrm{~cm}$ e $15,0 \mathrm{~cm}$ de espessura, e as paredes brasileiras, compostas de blocos de $11,5 \mathrm{~cm}$ e $14,0 \mathrm{~cm}$ de espessura.

A Tabela1 apresenta as características dos tijolos e blocos, dos Grupos I e II, utilizados nas edificações avaliadas.

\section{Critérios de avaliação}

Os critérios de desempenho acústico de cada país foram a base para a avaliação acústica das paredes. No Brasil, a norma de desempenho NBR 15575 (ABNT, 2008), que entrará em vigor em 2012, apresenta três níveis de desempenho, nomeadamente mínimo (M), intermediário (I) e superior (S). Embora apresente os três níveis de desempenho, somente o nível $\mathrm{M}$ é o exigido. Os demais são apresentados no anexo da norma e são apenas recomendações. A Tabela 2, extraída da parte 4 da norma acima mencionada, apresenta os valores de isolamento para os diferentes níveis de desempenho.

\begin{tabular}{c|c|c|c|c}
\hline \multirow{2}{*}{ Especificação } & \multicolumn{2}{|c|}{ Grupo I } & \multicolumn{2}{c}{ Grupo II } \\
\cline { 2 - 5 } & Brasil & Portugal & Brasil & Portugal \\
\hline $\begin{array}{c}\text { Dimensão do bloco/tijolo: } \\
\text { largura (cm) x altura (cm) } \\
\text { comprimento (cm) }\end{array}$ & $\begin{array}{c}11,5 \times 19,0 \times \\
39,0\end{array}$ & $\begin{array}{c}11,0 \times 20,0 \times \\
30,0\end{array}$ & $\begin{array}{c}14,0 \times 19,0 \times \\
39,0\end{array}$ & $\begin{array}{c}15,0 \times 20,0 \times \\
30,0\end{array}$ \\
\hline Massa média (kg) & 6,165 & 3,884 & 7,000 & 5,175 \\
\hline $\begin{array}{c}\text { Reboco de argamassa (cm) } \\
\text { (em cada face) }\end{array}$ & 1,50 & 2,00 & 1,50 & 2,00 \\
\hline $\begin{array}{c}\text { Espessura final da parede } \\
\text { (cm) }\end{array}$ & 14,5 & 15,0 & 17,0 & 19,0 \\
\hline
\end{tabular}

Tabela 1 - Características das paredes avaliadas 


\begin{tabular}{c|c|c|c}
\hline \multirow{2}{*}{ Elemento } & $\mathbf{D}_{\mathbf{n T}, \mathbf{w}}(\mathbf{d B})$ & $\begin{array}{c}\text { Índice de redução } \\
\text { sonora ponderado } \\
\mathbf{R}_{\mathbf{w}}(\mathbf{d B})\end{array}$ & $\begin{array}{c}\text { Nível de } \\
\text { desempenho }\end{array}$ \\
\hline Parede entre unidades & 40 a 44 & 45 a 49 & $\mathrm{M}$ \\
\cline { 2 - 4 } & 45 a 49 & 50 a 54 & $\mathrm{I}$ \\
\cline { 2 - 4 } $\begin{array}{c}\text { habitacionais autônomas } \\
\text { (parede de geminação) }\end{array}$ & $\geq 50$ & $\geq 55$ & $\mathrm{~S}$ \\
\hline
\end{tabular}

Fonte: NBR 15575-4 (ABNT, 2008).

Tabela 2 - Diferença padronizada de nível ponderada entre ambientes $\left(D_{n T, w}\right)$ para ensaio de campo e índice de redução sonora ponderado dos elementos construtivos $\left(R_{w}\right)$ para ensaio de laboratório

Em Portugal, não é uma norma que define os critérios para o desempenho dos edifícios, e sim um decreto-lei, que foi atualizado em 2008 e que teve seu parâmetro de avaliação até então utilizado, diferença normalizada de nível ponderada, $\mathrm{D}_{\mathrm{n}, \mathrm{w}}$, substituído pela diferença padronizada de nível ponderada, $\mathrm{D}_{\mathrm{nT}, \mathrm{w}}$, mesmo parâmetro utilizado pelo Brasil.

O critério português faz referência a um único valor de desempenho, e não a diferentes níveis de desempenho, como a norma brasileira. $\mathrm{O}$ artigo $5^{\circ}$, que trata dos edifícios habitacionais e mistos, e unidades hoteleiras, do Capítulo II, referente aos requisitos acústicos dos edifícios do Regulamento dos Requisitos Acústicos dos Edifícios, apresentado no Decreto-lei $n^{\text {o }} 96 / 2008$ do Ministério do Ambiente e do Ordenamento do Território, estabelece que o índice de isolamento sonoro entre ambientes internos e entre unidades habitacionais deverá ser, no mínimo, $\mathrm{D}_{\mathrm{nT}, \mathrm{w}}=50 \mathrm{~dB}$ (PORTUGAL, 2008).

Verifica-se que o critério português exige o nível de desempenho superior ao da norma brasileira. Porém, há que salientar que os edifícios portugueses requerem melhor vedação para ter o conforto térmico adequado durante o inverno. Dessa forma, o nível de conforto acústico passa a ser uma consequência dessa vedação, para atender às questões térmicas.

\section{Resultados e análise}

Os resultados e análises das medições estão apresentados a seguir. A identificação das paredes foi feita por meio dos blocos ou tijolos que as compõem, porém há que salientar que os resultados obtidos, tanto em campo quanto em laboratório, referem-se a todo o sistema construtivo.

\section{Desempenho acústico}

Os dois grupos de paredes foram avaliados acusticamente em laboratório. Em Portugal, o teste de isolação da parede foi realizado por Pião (2007). Os resultados estão disponibilizados em dissertação de mestrado e são utilizados neste trabalho, para efeito de comparação. A montagem da parede para a realização do teste no Brasil teve monitoramento direto da primeira autora deste trabalho. Houve o cuidado para que as juntas fossem cuidadosamente preenchidas e para que a colocação da argamassa por toda a área da parede, em ambas as faces, estivesse da forma o mais uniforme possível. Para evitar que houvesse transmissão sonora marginal, o corpo de prova (parede) foi isolado do restante da estrutura das câmaras reverberantes do laboratório por meio de câmaras pneumáticas. Esse procedimento é rotineiro no laboratório.

As Figuras 2 e 3 apresentam os resultados do índice de redução sonora em função da frequência obtidos nos testes de laboratório, das paredes de ambos os grupos, realizados no Brasil e em Portugal. 


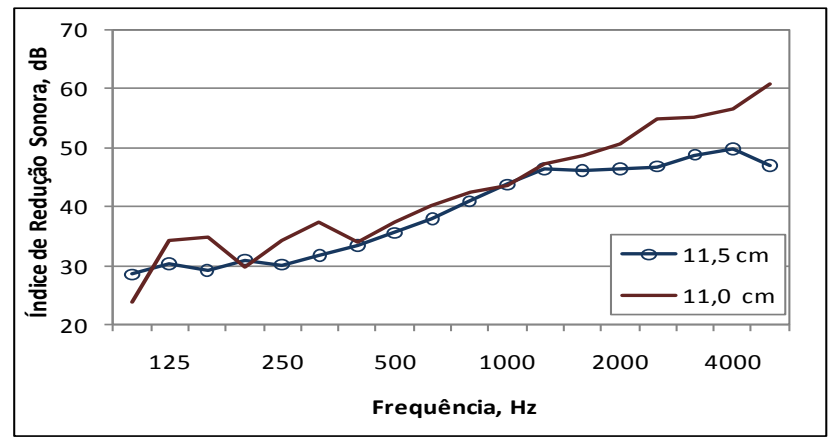

Obs.: espessuras: 11,5 cm (Brasil) e 11,0 cm (Portugal) (NETO, 2009).

Figura 2 - Desempenho em laboratório das paredes do Grupo I

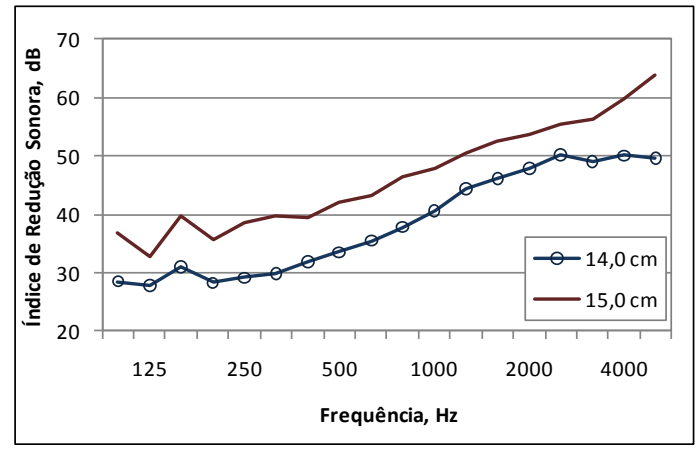

Obs.: espessuras: 14,0 cm (Brasil) (NETO, 2009) e 15,0 cm (Portugal) (PIÃO, 2007).

Figura 3 - Desempenho em laboratório das paredes do Grupo II

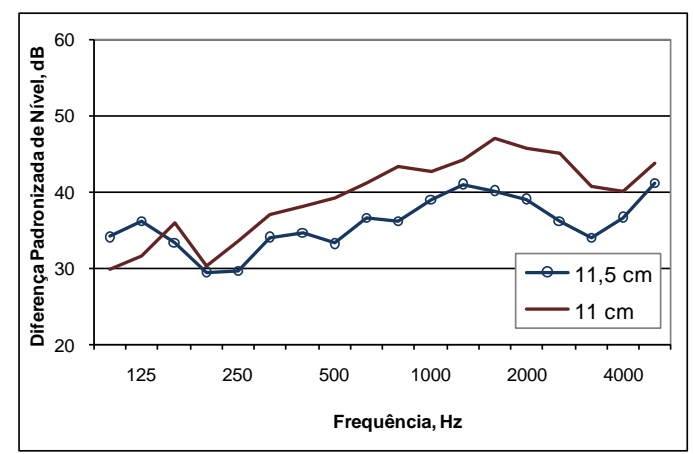

Obs.: espessuras: 11,5 cm (Brasil) e 11,0 cm (Portugal) (NETO, 2009).

Figura 4 - Desempenho em campo das paredes do Grupo I

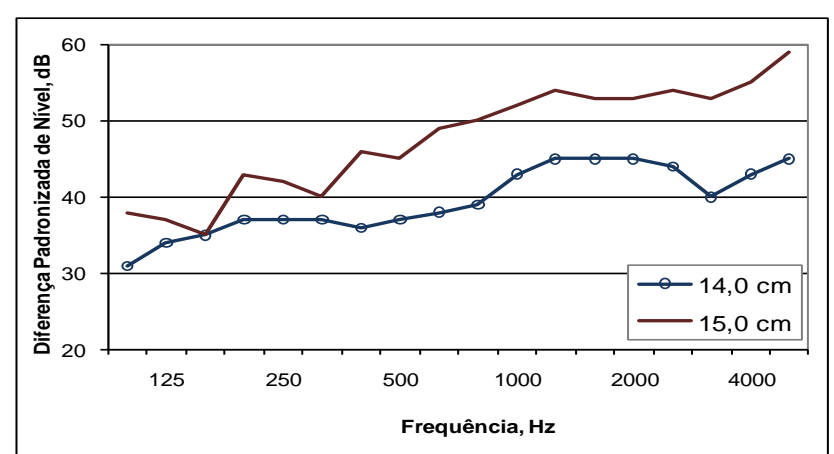

Obs.: espessuras: 14,0 cm (Brasil) e 15,0 cm (Portugal) (NETO, 2009).

Figura 5 - Desempenho em campo das paredes do Grupo II 


\begin{tabular}{c|c|c|c|c}
\hline Resultados dos testes & \multicolumn{2}{|c|}{ Grupo I } & \multicolumn{2}{c}{ Grupo II } \\
\hline & Brasil - 11,5 cm & Portugal - 11,0 cm & Brasil - 14,0 cm & Portugal - 15,0 cm \\
\hline Laboratório $\mathbf{R}_{\mathrm{w}}(\mathbf{d B})$ & $37 \boldsymbol{x}$ & $43^{8}$ & $39 \boldsymbol{X}$ & $47^{9}$ \\
\hline Campo $\mathrm{D}_{\mathrm{nT}, \mathrm{w}}(\mathbf{d B})$ & $37 \boldsymbol{x}$ & $42 \boldsymbol{x}$ & $41 \boldsymbol{V}$ & $50 \mathrm{~V}$ \\
\hline
\end{tabular}

Obs.: $(\boldsymbol{X})$ e $(\boldsymbol{V})$ representam o resultado da avaliação de atendimento aos critérios de desempenho de cada país.

Tabela 3 - Resumo dos resultados obtidos em laboratório e em campo

Em campo, a avaliação de desempenho das duas paredes foi realizada pelas autoras, tanto no Brasil quanto em Portugal. Nas Figuras 4 e 5, são apresentados os resultados da diferença padronizada de nível em função da frequência das paredes do Grupo I e do Grupo II respectivamente.

Os resultados dos testes realizados em laboratório e em campo estão apresentados na Tabela 3, juntamente com a avaliação quanto ao atendimento dos critérios de desempenho de cada país, nomeadamente NBR 15575 (ABNT, 2008) para o Brasil e Decreto-lei n ${ }^{\circ}$ 96/2008 para Portugal.

Os resultados dos testes em campo das paredes relativas ao Grupo I não atenderam aos critérios de seu respectivo país. As paredes do Grupo II atenderam aos respectivos critérios em campo. A parede brasileira atende ao critério da norma com nível de desempenho mínimo. Em laboratório, as paredes brasileiras, dos dois grupos, não atenderam ao critério.

Esperava-se que, em campo, os valores de isolamento fossem menores em $5 \mathrm{~dB}$ do que os valores em laboratório; somente as paredes portuguesas do Grupo I apresentaram resultado em laboratório superior ao resultado em campo. Isso porque, com base em alguns estudos de desempenho de paredes no Brasil e principalmente em documentos internacionais que estabelecem critérios de desempenho de edifícios, a norma NBR 15575 (ABNT, 2008) define que o desempenho em campo deve ser $5 \mathrm{~dB}$ inferior ao desempenho em laboratório (NETO; BERTOLI; BARRY, 2010). Ainda assim, a diferença de 1,0 $\mathrm{dB}$ entre esses resultados está dentro da variação esperada para testes em campo, que é de 2,0 dB. As duas paredes brasileiras desse mesmo grupo resultaram no mesmo valor absoluto de desempenho acústico, tanto em laboratório quanto em campo. Os resultados das paredes do Grupo II, tanto do Brasil quanto de Portugal, apresentaram valores superiores em campo do que em laboratório ( $2 \mathrm{~dB}$ e $3 \mathrm{~dB}$ respectivamente).

Inicialmente, esperava-se um melhor desempenho da parede de $11,5 \mathrm{~cm}$ ou, no mínimo, desempenho similar de isolamento em relação à parede de 11,0 cm em laboratório, visto ser a condição ideal para a avaliação da parede em si e a espessura final dos corpos de prova (paredes com argamassa) estar muito parecida, em ambos os países, o que mostra que a espessura não é o único parâmetro que justifica o desempenho, mas o sistema construtivo como um todo.

Deve-se salientar que em medições em laboratório, onde é obtido o desempenho somente do corpo de prova, por ser posicionado isoladamente da estrutura das câmaras reverberantes, o tijolo/bloco cerâmico em si não é o único responsável pelos valores de desempenho acústico. Contribuem para o desempenho também o tipo e a densidade de argamassa, a colocação da argamassa nas juntas e o próprio reboco. As paredes avaliadas nos testes em laboratório, tanto no Brasil quanto em Portugal, tiveram as juntas verticais e horizontais preenchidas com a argamassa de cimento. No Brasil, a colocação da argamassa durante a construção da parede é feita com o auxílio de bisnaga, como se pode verificar na Figura 6. Em Portugal, a pá de pedreiro é utilizada para a colocação da argamassa, espalhando-a por toda a face do tijolo, como se verifica na Figura 7. Entretanto, a diferença na espessura do reboco não aparenta ser significativa, de forma que, sozinha, justifique a diferença de $6 \mathrm{~dB}$ e $5 \mathrm{~dB}$ respectivamente entre os testes em laboratório e testes em campo do Grupo I.

A diferença na densidade da argamassa utilizada nas paredes poderia ser um dos fatores que pudesse influenciar os resultados do desempenho. Informações de Pião (2007) indicaram que a densidade volumétrica da argamassa utilizada em Portugal é de aproximadamente $2.050 \mathrm{~kg} / \mathrm{m} 3$. Esse valor está dentro do intervalo de $1.970 \mathrm{~kg} / \mathrm{m} 3$ a $2.290 \mathrm{~kg} / \mathrm{m} 3$, referente à densidade volumétrica de argamassa utilizada no Brasil.

Em trabalho publicado por Neto, Bertoli e Barry (2010), a influência da argamassa como reboco foi considerada não significativa em avaliações em campo em paredes compostas de blocos de concreto e cerâmico, rebocados em gesso e argamassa.

\footnotetext{
${ }^{8}$ Segundo o Decreto-lei n $96 / 2008$, não há avaliação do desempenho em laboratório.

${ }^{9}$ Idem.
} 


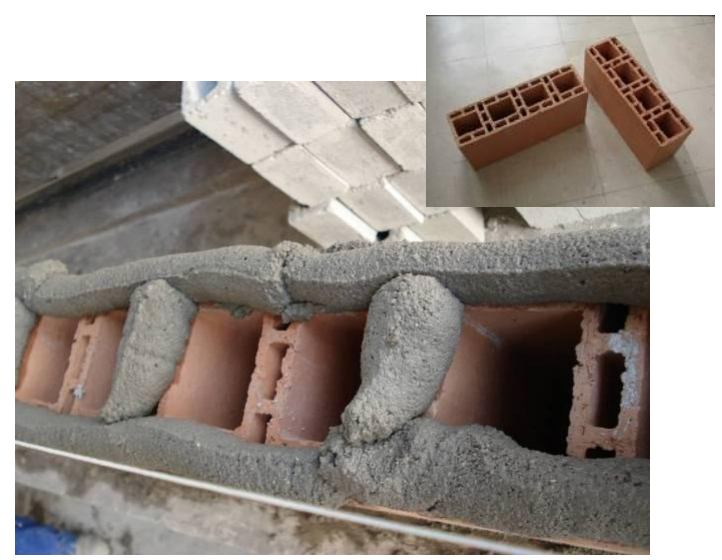

Figura 6 - Construção de parede de bloco cerâmico no Brasil, com o uso de bisnaga - no detalhe, peças de bloco cerâmico

Fonte: Associação... (2000).

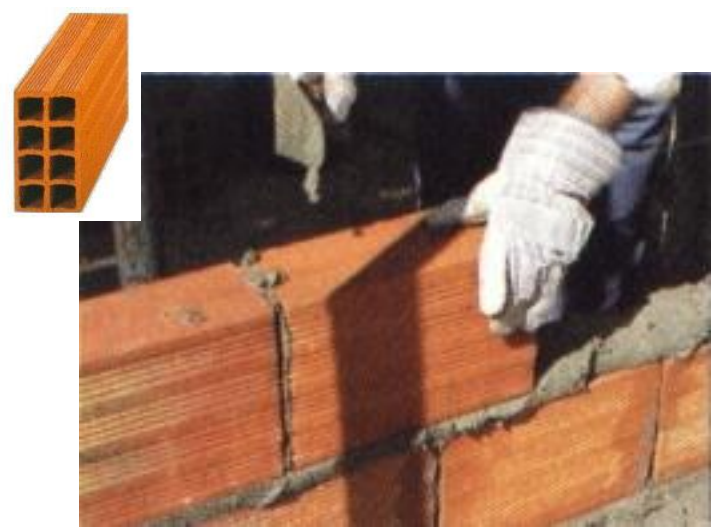

Figura 7 - Construção de parede de tijolo cerâmico em Portugal, com o uso da pá de pedreiro - no detalhe, uma peça de tijolo cerâmico

A Lei das Massas é usualmente a primeira justificativa para os resultados de desempenho. Embora os blocos brasileiros tenham mais massa do que os tijolos portugueses, o conjunto final de paredes de cada um dos dois grupos apresentou valor de densidade superficial muito próximo. Portanto, a Lei das Massas somente não justifica as diferenças nos resultados, ademais, segundo Paixão (2002), a Lei das Massas resulta de simplificações e, portanto, não pode ser aplicada indiscriminadamente na construção civil. A justificativa dos resultados, porém, depende de fatores que vão além do tijolo, do bloco ou do reboco. Entre esses fatores está a colocação da argamassa, a existência de orifícios e de caixas de luz, a estrutura do edifício e, ainda, a rigidez da parede, o amortecimento interno, a frequência crítica, o efeito de coincidência e a ressonância (BISTAFA, 2006; PAIXÃO, 2002). Cada material apresenta uma frequência crítica (da alvenaria com o reboco de argamassa está em torno de $200 \mathrm{~Hz}$ ), a partir da qual outros fatores passam a ser responsáveis pelo desempenho, em detrimento da massa. No caso da alvenaria, por ser um material composto, suas propriedades dependem das características individuais dos elementos que a constituem (PAIXÃO, 2002).

\section{Percepção do júri}

A Tabela 4 apresenta os resultados do percentual da percepção das sentenças entendidas pelo júri no teste subjetivo.

Verifica-se, pelos resultados apresentados na Tabela 4, que as paredes de ambos os grupos apresentam comportamentos similares, proporcionando maior privacidade para a voz masculina. Embora o desempenho das paredes seja maior para frequências maiores, a análise do ambiente de recepção por meio de parâmetros psicoacústicos como EDT (early decay time) e $\mathrm{D}_{50}$ (definition) se faz necessária. Adicionalmente, outros parâmetros que caracterizam o ambiente de recepção, como o tempo de reverberação e o ruído de fundo, também podem ser importantes para essa justificativa. Tal análise sobre sistemas construtivos similares está apresentada detalhadamente no trabalho de Neto (2009). Quanto à voz feminina, o comportamento de ambos os grupos também é similar, porque, embora possa ser mais ouvida em comparação com a voz masculina, a percepção se faz parcial e com dificuldade. 


\begin{tabular}{l|c|c|c|c|c|c|c|c}
\hline \multirow{2}{*}{$\begin{array}{l}\text { Percepção das } \\
\text { sentenças }\end{array}$} & \multicolumn{4}{|c|}{ Grupo I } & \multicolumn{4}{c}{ Grupo II } \\
\cline { 2 - 9 } & \multicolumn{2}{|c|}{ Brasil - 11,5 cm } & \multicolumn{2}{c|}{ Portugal - 11,0 cm } & \multicolumn{2}{c}{ Brasil - 14,0 cm } & \multicolumn{2}{c}{ Portugal - 15,0 cm } \\
\cline { 2 - 9 } & Fem. (\%) & $\begin{array}{c}\text { Masc. } \\
(\%)\end{array}$ & Fem. (\%) & $\begin{array}{c}\text { Masc. } \\
(\%)\end{array}$ & $\begin{array}{c}\text { Fem. } \\
(\%)\end{array}$ & $\begin{array}{c}\text { Masc. } \\
(\%)\end{array}$ & $\begin{array}{c}\text { Fem. } \\
(\%)\end{array}$ & Masc. (\%) \\
\hline $\begin{array}{l}\text { Ouve e entende } \\
\text { tudo com } \\
\text { facilidade }\end{array}$ & 0,0 & 0,0 & 0,0 & 0,0 & 0,0 & 0,0 & 0,0 & 0,0 \\
\hline $\begin{array}{l}\text { Ouve e entende } \\
\text { uma parte com } \\
\text { dificuldade }\end{array}$ & 13,1 & 9,2 & 18,3 & 0,0 & 31,5 & 16,2 & 35,0 & 21,7 \\
\hline $\begin{array}{l}\text { Ouve, mas não } \\
\text { entende }\end{array}$ & 86,9 & 90,8 & 81,7 & 98,3 & 68,5 & 83,8 & 65,0 & 78,3 \\
\hline Não ouve & 0,0 & 0,0 & 0,0 & 1,7 & 0,0 & 0,0 & 0,0 & 0,0 \\
\hline
\end{tabular}

Tabela 4 - Percentual da percepção das sentenças em teste subjetivo com o júri

Os valores da opção "ouve e entende uma parte com dificuldade" para o Grupo I, para ambas as vozes, estão próximos, com diferença aproximada de $5 \%$ para a voz feminina e de $9 \%$ para a voz masculina. E, justamente para a voz masculina, o menor valor dessa opção correspondeu à parede com melhor desempenho. No conjunto das vozes, feminina e masculina, o júri do Grupo I confirmou a menor percepção das sentenças com a parede de melhor desempenho.

Para a mesma opção "ouve e entende uma parte com dificuldade", as paredes do Grupo II apresentam a diferença aproximada de $4 \%$ para a voz feminina e de $5 \%$ para a voz masculina, que são os mesmos valores, aproximados, para a opção "ouve, mas não entende". O resultado com o júri do Grupo II não foi confirmado com o desempenho da parede; segundo o júri, a parede com pior desempenho proporciona melhor privacidade em relação à percepção da conversação.

Do ponto de vista do júri, a diferença na percepção das sentenças se faz maior com as paredes do Grupo I do que com as paredes do Grupo II, embora a diferença no desempenho em campo das paredes do Grupo II se faz maior do que a do Grupo I.

O júri do Grupo II surpreendeu com o resultado de que a parede com pior desempenho proporciona melhor privacidade. A percepção das sentenças por meio de um júri deve ser mais bem avaliada porque somente o desempenho da parede não demonstra ser preponderante para a percepção. Outros fatores, como o tempo de reverberação no ambiente onde é feita a emissão das sentenças e no ambiente onde está o júri, a sensação da reverberação do ambiente, dada pelo EDT (early decay time), a definição, dada por $\mathrm{D}_{50}$, auxiliam nessa análise. Esses e outros parâmetros psicoacústicos que influenciam na percepção da palavra falada foram estudados por Neto (2009), que mostra que as condições acústicas do ambiente de recepção devem ser adequadas para que haja melhor inteligibilidade das sentenças emitidas no ambiente adjacente e que o desempenho acústico da parede não é fator único na percepção da fala. O tempo de reverberação e o ruído de fundo no ambiente de recepção, como foi mencionado, são fatores que influenciam diretamente a percepção da fala, independentemente de onde esteja a fonte sonora: no mesmo ambiente de recepção, no ambiente adjacente ou em ambiente próximo. Ambientes com maior reverberação ou ruído de fundo podem mascarar as sentenças, dificultando a percepção, dando a sensação de maior privacidade, mesmo com desempenho não tão bom da parede entre os ambientes de emissão e recepção. A sensação de reverberação maior ou menor causada pelo ambiente, e avaliada por meio de EDT em faixas específicas de frequências, também pode contribuir com a maior ou a menor percepção das sentenças e, de modo especial, pronunciadas por voz feminina ou masculina. A definição, $D_{50}$, também analisada por faixa de frequência mostra em quais ambientes a percepção da voz masculina ou feminina é melhor.

\section{Conclusões}

Foram apresentados neste trabalho resultados do desempenho com medições objetivas em laboratório e em campo, e resultados de desempenho com avaliação subjetiva, com o júri.

Em relação às paredes do Grupo I, esperava-se, com base na Lei das Massas, que, pela diferença de espessura, a parede composta de tijolo cerâmico de $11,5 \mathrm{~cm}$ deveria apresentar melhor desempenho do que a composta de tijolo cerâmico de $11,0 \mathrm{~cm}$. Porém, os resultados mostraram o contrário, indicando que a espessura não é o único parâmetro que justifique o desempenho, mas o sistema construtivo como um todo. Por outro ângulo, a parede de espessura final de $15,0 \mathrm{~cm}$ deveria 
apresentar melhor desempenho acústico do que a de $14,5 \mathrm{~cm}$, e é o que ocorreu. Verifica-se, então, que, além do tijolo ou bloco e do reboco, a montagem da parede com a argamassa colocada nas juntas, vertical e horizontal, contribui fortemente para o desempenho acústico da parede. A diferença final na espessura é aparentemente pequena para uma diferença grande no valor final do desempenho. Ambas as paredes apresentam densidade superficial similar, porém a Lei das Massas, nesse caso, não é a melhor justificativa para a diferença no desempenho. Como foi mencionado no decorrer do trabalho, há outros fatores que influenciam no desempenho além da massa, como rigidez, amortecimento interno, frequência crítica e ressonância. $O$ fato de $\mathrm{o}$ resultado do cálculo do desempenho pela Lei das Massas não corresponder aos resultados experimentais corrobora tal análise.

O conforto acústico, proporcionado pela redução da inteligibilidade da fala, nos ambientes adjacentes a essa parede é consequência desse desempenho, visto que o júri confirmou a menor percepção das sentenças com a parede de maior valor da diferença padronizada de nível ponderado. Salienta-se, entretanto, que a análise dos resultados obtidos com o júri pode ser complementada por meio de parâmetros psicoacústicos, como EDT (early decay time) e $\mathrm{D}_{50}$ (definition), e outros parâmetros que caracterizam o ambiente de recepção, como o tempo de reverberação e o ruído de fundo.

Quanto às paredes do Grupo II, a parede portuguesa apresentou valor de desempenho muito superior à parede brasileira. Da mesma forma como no Grupo I, o bloco brasileiro, embora mais pesado do que o tijolo português, não foi o fator significativo sobre o resultado. A densidade superficial de ambas as paredes também apresentava valores muito próximos como nas paredes do Grupo I. Entretanto, a espessura da parede portuguesa, sendo superior em $2,0 \mathrm{~cm}$ sobre a brasileira, pode justificar a diferença no valor do desempenho (embora seja possível, não há resultados suficientes para garantir), adicionalmente ao cuidado no preenchimento das juntas, colocação do reboco e outros cuidados já mencionados.

$\mathrm{Na}$ avaliação das paredes do Grupo II, o júri contradisse que o melhor desempenho proporciona melhor privacidade. Verifica-se, então, que somente o desempenho da parede não é fator preponderante na percepção das sentenças. Há outros fatores que, além do desempenho, influenciam a diferença nos resultados entre as paredes do Grupo I e do Grupo II, como o EDT (early decay time) e $\mathrm{D}_{50}$ (definition), adicionalmente ao tempo de reverberação e ao ruído de fundo no ambiente de recepção.

$\mathrm{E}$, finalmente, em relação aos critérios de desempenho, a parede portuguesa do Grupo I poderia ser utilizada como parede de geminação no Brasil, porém com a classificação de desempenho mínimo. A parede portuguesa do Grupo II teria classificação superior no nível de desempenho, se pudesse ser aplicada no Brasil. Já a parede brasileira do Grupo I não poderia ser utilizada no Brasil e menos ainda em Portugal, onde os critérios de desempenho são mais rigorosos, como parede de geminação. A parede brasileira do Grupo II é classificada com nível mínimo de desempenho no Brasil. O fato de nenhuma das paredes brasileiras atender ao critério em laboratório sugere que haja maior número de medições e, permanecendo tais resultados, deve-se propor uma revisão da norma de desempenho NBR 15575 (ABNT, 2008).

\section{Referências}

\section{ASSOCIAÇÃO BRASILEIRA DE NORMAS}

TÉCNICAS. NBR 15575-4: edifícios

habitacionais de até cinco pavimentos: desempenho: parte 4: requisitos para sistemas de vedações internas e externas. Rio de Janeiro. 2008.

ASSOCIAÇÃO BRASILEIRA DA
CONSTRUÇÃO INDUSTRIALIZADA. Manual
Técnico de Alvenaria. São Paulo:
ABCI/PROJETO, 1990. 280 p.

ASSOCIAÇÃO PORTUGUESA DA INDÚSTRIA DE CERÂMICA (APICER). Manual de Alvenaria de Tijolo. Coimbra: APICER, 2000. 208 p.

BARRY, Peter J. Desempenho Acústico em Edifícios: grandezas, métodos, normas e critérios. In: SEMINÁRIO HABITAÇÃO: DESEMPENHO E INOVAÇÃO TECNOLÓGICA, 2005, São Paulo. Anais... São Paulo: IPT, 2005. p. 76-83.

BARRY, Peter. Desempenho Acústico em Edifícios: grandezas, métodos, normas e critérios. In: SEMINÁRIO DE ACÚSTICA ARQUITETÔNICA CONTEMPORÂNEA, 4., 2008, São Paulo. Anais... São Paulo, 2008.

CARVANAUGH, W. J. et al. Speech Privacy in Buildings. Acoustical Society of America, v. 34, n. 4, p. 475-492, 1962.

COLLINS, R. E. et al. Vacuum Glazing: a new component for insulating windows. Building and Environment, v. 30, n. 4, p. 459-492, 1995. 
CROOME, D. J. Noise, Building and People. Great Britain: Pergamon Press, 1977. v. 11, $613 \mathrm{p}$.

BARING, João Gualberto de Azevedo. Isolação Sonora de Paredes e Divisórias. In: Instituto de Pessquisas Tecnológicas; PINI. Tecnologia de Edificações. São Paulo. PINI/ IPT, 1988. p. 429-434.

BISTAFA, Sylvio R. Acústica Aplicada ao Controle do Ruído. São Paulo: Edgard Blücher, 2006. 368 p.

FUCHS, H. V. et al. Creating Low-Noise Environments in Communication Room Applied Acoustics, v. 62, n. 12, p. 1375-1396, dez. 2001.

GAMBINI, Caroline. Reconhecimento de Fala em Escolares Com e Sem Prática Musical e Diferentes Níveis Sócio-Culturais. 2006. 43 f. Monografia (Especialização) - Universidade Federal de Santa Maria, Santa Maria, 2006.

GERRETSEN, Eddy. Sound Insulation Quality in Dutch Dwellings. In: DAGA, 2003, Aachen. Proceedings... Aachen: Institut für Technische Akustik der RWTH, 2003a.

GERRETSEN, Eddy. Prediction of Sound Insulation in Buildings: a tool to improve the acoustic quality. In: DAGA, 2003, Aachen. Proceedings... Aachen: Institut für Technische Akustik der RWTH, 2003b.

GRIMWOOD, Colin. Complaints About Poor Sound Insulation Between Dwellings in England and Wales. Applied Acoustics, Great Britain, v. 52, n. 3/4, p. 211-223, nov./dez. 1997.

HASSAL, J. R.; ZAVERI, K. Acoustic Noise Mmeasurements. 5. ed. Dinamarca: K. Larsen and Son, 1988. 310 p.

INTERNATIONAL ORGANIZATION FOR STANDARDIZATION. ISO 140-3: acoustics: measurement of sound insulation in buildings and of building elements: part 3: laboratory measurements of airborne sound insulation of building elements. Genève, 1995.

INTERNATIONAL ORGANIZATION FOR STANDARDIZATION. ISO 140-4:

acoustics: measurement of sound insulation in buildings and of building elements: part 4: field measurements of airborne sound insulation between rooms. Genève, 1998.

INTERNATIONAL ORGANIZATION FOR STANDARDIZATION. ISO 354: acoustics: measurement of sound absorption in a reverberation room. Genève, 2003.
INTERNATIONAL ORGANIZATION FOR

STANDARDIZATION. ISO 717-1:

acoustics: rating of sound insulation in buildings and of building elements: part 1: airborne sound insulation. Genève, 1996.

LAHTELA, Tero. Sound Insulation: guidelines for wooden residential buildings. Finlândia: Wood Focus, 2005. 114 p.

NETO, Maria de Fatima Ferreira. Nível de Conforto Acústico: uma proposta para edifícios residenciais. 2009. 257 f. Tese (Doutorado em Engenharia Civil) - Programa de Pós-Graduação em Engenharia Civil, Universidade Estadual de Campinas, Campinas, 2009.

NETO, Maria de Fatima F.; BERTOLI, Stelamaris Rolla; BARRY, Peter J. Diferença Entre Testes de Desempenho Acústico em Laboratório e Campo em Paredes de Alvenaria. In: ENCONTRO DA SOCIEDADE BRASILEIRA DE ACÚSTICA, 23., 2010, Salvador. Anais... Salvador: SOBRAC, 2010.

PAIXÃO, Dinara Xavier. Caracterização do Isolamento Acústico de Uma Parede de Alvenaria Utilizando Análise Estatística de Energia (SEA). 2002. 182 f. Tese (Doutorado em Engenharia de Produção) - Programa de PósGraduação em Engenharia de Produção, Universidade Federal de Santa Catarina, Florianópolis, 2002.

PIÃO, Adriano Fresco das Neves Simões. Isolamento Acústico de Paredes de Alvenaria de Tijolo: resultados experimentais versus modelos numéricos. Coimbra, 2007.140 f. Dissertação (Mestrado em Engenharia Civil) - Departamento de Engenharia Civil, Faculdade de Ciências e Tecnologia, Universidade de Coimbra, Coimbra, 2007.

PORTUGAL. Decreto-Lei no 96/2008.

Regulamento dos Requisitos Acústicos dos Edifícios. Diário da República I-Série A. nº 110, p. 3359-3372. Lisboa. 2008.

UTLEY, W. A.; BULLER, I. B. A Study of Complaints About Noise from Domestic Premises. Journal of Sound and Vibration, v. 127, n. 2, p. 319-330, dez. 1988.

\section{Agradecimentos}

As autoras agradecem ao Departamento de Engenharia Civil da Faculdade de Ciências e Tecnologia da Universidade de Coimbra, nas pessoas da Prof. a Dr. a Julieta António e do Prof. Dr. Luis Godinho, pelo empréstimo dos equipamentos necessários para a realização das medições em Portugal. 